\title{
Icosapent Ethyl (Eicosapentaenoic Acid Ethyl Ester): Effects Upon High-Sensitivity C-Reactive Protein and Lipid Parameters in Patients With Metabolic Syndrome
}

\author{
Harold E. Bays, MD, ${ }^{1}$ Christie M. Ballantyne, MD, ${ }^{2}$ Rene A. Braeckman, PhD, William G. Stirtan PhD, ${ }^{4}$ \\ Ralph T. Doyle Jr, BA, ${ }^{4}$ Sephy Philip, PharmD, ${ }^{4,5}$ Paresh N. Soni, MD, PhD, ${ }^{6}$ and Rebecca A. Juliano, $\mathrm{PhD}^{4}$
}

\begin{abstract}
Background: The aim of this analysis was to examine the effects of icosapent ethyl (eicosapentaenoic acid ethyl ester, IPE) on high-sensitivity C-reactive protein (hsCRP) and lipid parameters in patients with metabolic syndrome, with and without stable statin therapy.

Methods: This post hoc exploratory analysis evaluated patients with metabolic syndrome treated with IPE 4 grams/day, IPE 2 grams/day, or placebo in phase 3, randomized, placebo-controlled studies entitled: MARINE [triglyceride (TG) levels $\geq 500$ and $\leq 2000 \mathrm{mg} / \mathrm{dL}$ ] and ANCHOR [TG levels $\geq 200$ and $<500 \mathrm{mg} / \mathrm{dL}$, despite low-density lipoprotein cholesterol (LDL-C) control with stable statin therapy].

Results: Compared with placebo in patients with metabolic syndrome in MARINE $(n=204)$ and ANCHOR $(n=645)$, at the approved dose of 4 grams/day, IPE significantly lowered hsCRP levels $40.0 \%(P=0.0007)$ in MARINE and $23.0 \%(P=0.0003)$ in ANCHOR. Compared with placebo in MARINE, which included patients with and without statin therapy, IPE 4 grams/day significantly reduced hsCRP levels $78.0 \%$ in statin-treated patients $(P=0.0035, n=16)$. Compared with placebo in MARINE, IPE 4 grams/day significantly reduced TG levels $(35.0 \% ; P<0.0001)$, non-high-density lipoprotein cholesterol (non-HDL-C; $19.9 \% ; P<0.0001$ ), and apolipoprotein B levels (ApoB) $(9.1 \% ; P=0.0015)$ without raising LDL-C levels. Compared with placebo in ANCHOR, IPE 4 grams/day significantly reduced TG $(21.7 \% ; P<0.0001)$, non-HDL-C $(13.5 \% ; P<0.0001)$, ApoB $(8.8 \% ; P<0.0001)$, LDL-C $(5.2 \% ; P=0.0236)$, and HDL-C levels $(4.0 \% ; P=0.0053)$.

Conclusions: Compared with placebo, IPE 4 grams/day significantly lowered hsCRP levels and improved lipids without raising LDL-C levels in patients with metabolic syndrome and high ( $\geq 200$ and $<500 \mathrm{mg} / \mathrm{dL}$ ) or very high ( $\geq 500$ and $\leq 2000 \mathrm{mg} / \mathrm{dL}$ ) TG levels, with or without stable statin therapy.
\end{abstract}

\section{Introduction}

$\mathrm{M}$ ETABOLIC SYNDROME IS A constellation of cardiovascular disease (CVD) risk factors whose criteria include increased waist circumference (WC) as the only anatomic abnormality. An increase in WC is a surrogate measure of visceral adiposity, which, when increased, reflects total body fat immune and endocrine derangements (adiposopathy), as well as predisposition to common metabolic disturbances that all contribute to major CVD risk factors. ${ }^{1-3}$ The metabolic components of the metabolic syndrome include high blood pressure, hyperglycemia, and elevated triglyceride (TG) levels. ${ }^{4-8}$ Worldwide, the prevalence of metabolic syndrome is increasing, largely due to the increased prevalence of

\footnotetext{
${ }^{1}$ Louisville Metabolic and Atherosclerosis Research Center, Louisville, Kentucky.

${ }^{2}$ Baylor College of Medicine and the Methodist DeBakey Heart and Vascular Center, Houston, Texas.

${ }^{3}$ Doylestown, Pennsylvania.

${ }^{4}$ Amarin Pharma Inc., Bedminster, New Jersey.

${ }^{5}$ Chilton Medical Center, Pompton Plains, New Jersey.

${ }^{6}$ Mystic, Connecticut.
}

(C) Harold E. Bays, et al. 2015; Published by Mary Ann Liebert, Inc. This Open Access article is distributed under the terms of the Creative Commons Attribution Noncommercial License ( $<$ http://creativecommons.org/licenses/by-nc/4.0/ $>$ ) which permits any noncommercial use, distribution, and reproduction in any medium, provided the original author(s) and the source are credited. 
overweight, obesity, and sedentary lifestyles. ${ }^{9}$ Patients diagnosed with metabolic syndrome have a two-fold increased risk of developing CVD in the subsequent 5-10 years and a five-fold increased risk of developing type 2 diabetes mellitus (T2DM) compared with those without the metabolic syndrome. ${ }^{5}$

C-reactive protein (CRP) is a marker for inflammation. Its increase may be due to direct secretion from dysfunctional adipocytes, or, more commonly, due to an increase in hepatic secretion as the result of increased interleukin-6 (IL-6) from adiposopathic proinflammatory responses from adipose tissue. ${ }^{2,10,11}$ An increase in CRP levels may also reflect vascular inflammation, ${ }^{6}$ which is an integral component of the atherogenic process. ${ }^{12}$ Thus, whether it reflects an increase in an adiposopathic proinflammatory response from an increase in body fat (which also accompanies an increase in major CVD risk factors ${ }^{7}$ ) or due to direct responses from a proinflammatory and proatherogenic vasculature, an increase in CRP levels is a marker for increased CVD risk. ${ }^{13,14}$

Omega-3 fatty acids are polyunsaturated fatty acids with a double bond at the third carbon from the methyl (omega) end. ${ }^{15}$ Omega-3 fatty acids, including eicosapentaenoic acid (EPA) and docosahexaenoic acid (DHA), are generally considered essential fatty acids, in that the human body has a limited ability to create an unsaturated bond at the omega- 3 position. Thus, the majority of the body's EPA and DHA are derived from consumption, most commonly from marine fish. ${ }^{16}$ Because EPA and DHA are lipophilic, these omega- 3 fatty acids diffuse easily and are stored in body fat and lipid membranes. ${ }^{15}$ Thus, consumed omega-3 fatty acids are reflected by measuring omega-3 fatty acids in plasma, cellular membranes, or adipose tissue. ${ }^{17}$

EPA and DHA significantly reduce TG levels and may improve other CVD risk factors, ${ }^{18}$ including markers of inflammation. When combined, EPA and DHA may reduce some inflammatory markers such as lipoprotein-associated phospholipase $\mathrm{A}_{2} \cdot{ }^{19}$ EPA combined with DHA has more inconsistent effects upon high-sensitivity CRP (hsCRP). ${ }^{20-22}$ Conversely, EPA alone may decrease not only lipoproteinassociated phospholipase $\mathrm{A}_{2}$ levels, but also oxidized LDL and hsCRP levels. ${ }^{23-25}$

Icosapent ethyl (eicosapentaenoic acid ethyl ester, IPE; Vascepa ${ }^{\circledR}$, Amarin Pharma Inc., Bedminster, NJ) is a highpurity prescription form of EPA ethyl ester approved by the US Food and Drug Administration as an adjunct to diet to reduce TG levels in adult patients with severe $(\geq 500 \mathrm{mg} /$ dL) hypertriglyceridemia. ${ }^{26}$ Two randomized placebocontrolled studies demonstrated the efficacy of IPE in improving lipid parameters in patients with elevated TG levels. ${ }^{27,28}$ The pivotal MARINE study assessed the efficacy and safety of IPE in 229 patients with TG levels $\geq 500 \mathrm{mg} /$ $\mathrm{dL}$ and $\leq 2000 \mathrm{mg} / \mathrm{dL}^{27}$ The ANCHOR study evaluated the efficacy and safety of IPE in 702 patients with TG levels $\geq 200 \mathrm{mg} / \mathrm{dL}$ and $<500 \mathrm{mg} / \mathrm{dL}$ despite control of lowdensity lipoprotein cholesterol (LDL-C) levels with stable statin therapy. ${ }^{28}$ Compared with placebo in both studies, IPE 4 grams/day significantly reduced TG levels and other lipid parameters without raising LDL-C levels.

Given that metabolic syndrome is often considered an inflammatory state that links an increase in body fat to metabolic diseases such as $\mathrm{T} 2 \mathrm{DM},{ }^{29}$ that an increase in inflammatory factors such as CRP may also be an important marker of CVD risk, ${ }^{2,30,31}$ and that omega-3 fatty acids may have anti-inflammatory effects, this post hoc analysis of the MARINE and
ANCHOR studies examined the effects of IPE on hsCRP and lipid parameters in patients with metabolic syndrome (a potentially proinflammatory state) to determine the effects of IPE in these patients with and without stable statin therapy.

\section{Methods}

\section{Design and patients}

This post hoc analysis evaluated patients with metabolic syndrome at baseline from the MARINE and ANCHOR studies. $^{27,28}$ Briefly, both were phase 3, placebo-controlled, randomized, double-blind, multicenter studies in eligible patients aged $>18$ years. Both studies included a 4 - to 6-week initial stabilization period of diet and lifestyle changes and medication washout, a 2- to 3-week lipid-qualifying period, and a 12-week double-blind treatment period during which subjects received either IPE 4 grams/day, IPE 2 grams/day, or matched placebo. The MARINE study enrolled subjects with TG levels $\geq 500 \mathrm{mg} / \mathrm{dL}$ and $\leq 2000 \mathrm{mg} / \mathrm{dL}$; patients could continue receiving a stable statin regimen with or without ezetimibe if the dose was stable for $\geq 4$ weeks. The ANCHOR study was conducted in statin-treated subjects with high cardiovascular risk, well-controlled LDL-C $(\geq 40 \mathrm{mg} / \mathrm{dL}$ and $<115 \mathrm{mg} / \mathrm{dL}$ ), and residually high TG levels $(\geq 200$ and $<500 \mathrm{mg} / \mathrm{dL}$ ). Patients in the ANCHOR trial were required to be on a stable statin therapy of atorvastatin, rosuvastatin, or simvastatin with or without ezetimibe for $\geq 4$ weeks (at a stable dose such that the LDL-C qualifying criteria were met; switching between statins was prohibited). The studies were conducted in accordance with the principles of Good Clinical Practice and the Declaration of Helsinki. The protocol was approved by the appropriate institutional review boards, and patients underwent the informed consent process as documented by a written informed consent.

Patients were identified as having metabolic syndrome if they had three or more of the following at baseline: Body mass index $(\mathrm{BMI}) \geq 30 \mathrm{~kg} / \mathrm{m}^{2}$ (substituted for waist circumference); TG levels $\geq 150 \mathrm{mg} / \mathrm{dL}$; high-density lipoprotein cholesterol (HDL-C) levels $<40 \mathrm{mg} / \mathrm{dL}$ in men or $<50 \mathrm{mg} / \mathrm{dL}$ in women; systolic blood pressure (SBP) $\geq 130 \mathrm{mmHg}$ or diastolic blood pressure (DBP) $\geq 85 \mathrm{mmHg}$, or on antihypertensive medication; and fasting plasma glucose levels >100 mg/ $\mathrm{dL}$ or on antidiabetes medication. ${ }^{5,9}$

\section{End points and measurements}

The end points reported here include median percentage changes from baseline to week 12 in hsCRP, TG, LDL-C, non-HDL-C, apolipoprotein B (ApoB), and HDL-C levels compared with placebo. Measurements are presented herein in $\mathrm{mg} / \mathrm{dL}$, with the exception of hsCRP, which is reported in $\mathrm{mg} /$ L. To convert lipids from $\mathrm{mg} / \mathrm{dL}$ to the International System (SI) units of millimoles/liter (mmol/L), multiply TG levels by 0.0113 and multiply cholesterol levels by 0.0259 . To convert ApoB levels to the SI units of grams/L, multiply by 0.01 .

Immunonephelometry (Dade Behring BNII Nephelometer, Siemens Healthcare Diagnostics, Deerfield, IL) was used to measure hsCRP; other end points were measured using methods previously described. ${ }^{27}$ TGs and cholesterol were measured using enzymatic colorimetric tests (Olympus AU2700 or AU5400 Analyzer, Olympus, Center Valley, PA), and non-HDL-C was calculated by subtracting HDL-C from total cholesterol. 


\section{Statistical analysis}

Efficacy analyses were performed on the intent-to-treat (ITT) population, defined as all randomized patients who had a baseline efficacy measurement, received at least one dose of study drug, and had at least one post-baseline efficacy measurement.

Median differences between the IPE and placebo groups were estimated with the Hodges-Lehmann method. $P$ values are from the Wilcoxon rank-sum test. A $P$ value $\leq 0.05$ was the specified alpha for significance for exploratory end points in the MARINE and ANCHOR studies and was used in this post hoc analysis; no adjustments were made for multiple comparisons. Statistical analysis was performed with SAS software version 8.2 or higher (SAS Institute, Cary, NC).

\section{Results}

\section{Baseline characteristics}

In the MARINE study, 204 of the 229 enrolled patients (89.1\%) had metabolic syndrome at baseline (IPE 4 grams/ day, $n=65$; IPE 2 grams/day, $n=69$; placebo, $n=70$ ). Among these patients, 153 were not receiving statin therapy and 51 were receiving stable statin therapy. In the ANCHOR study, 645 of the 702 patients $(91.9 \%)$ had metabolic syndrome at baseline (IPE 4 grams/day, $n=211$; IPE 2 grams/ day, $n=219$; placebo, $n=215$ ). As noted before, per protocol, all ANCHOR participants received stable statin therapy prior to study entry.

\section{IPE effects on hsCRP in patients with metabolic syndrome}

Results from the MARINE study are shown in Table 1 and Fig. 1. Compared with placebo in patients with metabolic syndrome, IPE 4 grams/day significantly reduced hsCRP levels by $40.0 \%(P=0.0007)$. Compared with placebo, IPE 4 grams/day significantly reduced hsCRP levels by $27.6 \%$ $(P=0.0385)$ in patients not receiving statin therapy and by $78.0 \%(P=0.0035)$ in patients receiving stable statin therapy. Compared with placebo, reductions in hsCRP with IPE 2 grams/day did not achieve statistical significance.

Results from the ANCHOR study are shown in Table 2 and Fig. 2. Compared with placebo in patients with metabolic syndrome, IPE 4 grams/day significantly reduced hsCRP levels from baseline by $23.0 \%(P=0.0003)$. Reductions in hsCRP with IPE 2 grams/day did not achieve statistical significance.

\section{IPE effects on lipid end points in patients with metabolic syndrome}

Among those in the MARINE and ANCHOR studies with metabolic syndrome, IPE improved lipid levels. Compared with placebo in patients with metabolic syndrome from the MARINE study, IPE 4 grams/day significantly reduced TG (35.0\%; $P<0.0001)$, non-HDL-C $(19.9 \% ; P<0.0001)$, and ApoB levels $(9.1 \% ; P=0.0015)$, with no significant changes in LDL-C or HDL-C levels (Table 1 and Fig. 1). Compared with placebo in patients with metabolic syndrome from the ANCHOR study, IPE 4 grams/day reduced TG (21.7\%; $P<0.0001), \quad$ LDL-C $(5.2 \% ; \quad P=0.0236), \quad$ non-HDL-C $(13.5 \% ; P<0.0001)$, ApoB $(8.8 \% ; P<0.0001)$, and HDL-C levels (4.0\%; $P=0.0053$ ) (Table 2 and Fig. 2). Changes in these parameters with IPE 2 grams/day are also shown in Tables 1 and 2 and Figs. 1 and 2.

\section{Discussion}

This post hoc analysis from the MARINE and ANCHOR studies examined the effects of IPE on the inflammatory marker hsCRP, as well as the lipid parameters TGs, LDL-C, non-HDL-C, ApoB, and HDL-C in the subset of patients with metabolic syndrome. In patients with metabolic syndrome and high $(\geq 200 \mathrm{mg} / \mathrm{dL}$ and $<500 \mathrm{mg} / \mathrm{dL})$ or very high $(\geq 500 \mathrm{mg} / \mathrm{dL}$ and $\leq 2000 \mathrm{mg} / \mathrm{dL})$ TG levels, IPE 4 grams/day improved lipid parameters compared with placebo and reduced hsCRP levels, a known marker for CVD risk, without increasing LDL-C levels. The results for hsCRP were similar to those observed in a previous study of markers of inflammation in all patients from the MARINE and ANCHOR studies. ${ }^{23}$

Statin therapy has anti-inflammatory effects, as reflected by a reduction in elevated hsCRP levels. The Justification for the Use of Statins in Prevention: an Intervention Trial Evaluating Rosuvastatin (JUPITER) was a large CVD outcomes study of 17,802 "apparently healthy" subjects with LDL-C levels $\leq 130 \mathrm{mg} / \mathrm{dL}$ ( $3.4 \mathrm{mmol} / \mathrm{L})$, but no history of $\mathrm{CVD}^{32}$ An entry criterion included elevated hsCRP levels $\geq 2.0 \mathrm{mg} / \mathrm{L}$. Baseline median BMI was $28 \mathrm{~kg} / \mathrm{m}^{2}$, and metabolic syndrome was present in $41 \%$ of study participants. ${ }^{32}$ After a median follow-up of 1.9 years, rosuvastatin significantly reduced the incidence of major cardiovascular events. At 12 months, rosuvastatin reduced median hsCRP, LDL-C, and TG levels $37 \%, 50 \%$, and $17 \%$, respectively (all $P<0.001) .{ }^{32}$ Possible interpretations include a role for elevated hsCRP levels as not only a marker of vascular inflammation, but also as being directly involved in the pathogenesis of atherosclerosis and thrombosis. ${ }^{2}$ Alternatively, the high mean BMI, high percentage of study participants with metabolic syndrome, and the elevated hsCRP levels may suggest that the disease state of adiposopathy was present at baseline. ${ }^{2}$ Finally, a reduction in inflammatory markers (e.g., IL-6, hsCRP) with statins may, in part, be due to statin-induced reductions in adipose tissue inflammation. ${ }^{33,34}$

Similarly, in a study of 48 dyslipidemic men with visceral obesity treated with: (1) atorvastatin alone; (2) EPA + DHA; (3) atorvastatin +EPA + DHA; or (4) placebo, atorvastatin, and atorvastatin + EPA + DHA significantly decreased hsCRP and IL-6 levels. However, EPA + DHA alone did not reduce hsCRP levels. ${ }^{20}$ The potential for statin therapy to reduce hsCRP levels helped contribute to the study design for both MARINE and ANCHOR wherein statin-treated patients were required to be on stable statin doses prior to study entry. ${ }^{27,28}$ Thus, the baseline hsCRP level was determined after stabilization of statin therapy in the MARINE and ANCHOR studies, which helped mitigate the possibility that the changes in hsCRP levels were statin-induced.

As noted before, data on the effect of EPA and/or DHA on plasma CRP or hsCRP levels are inconsistent. $^{20-22,24,25,35-46} \mathrm{~A}$ cross-sectional study in 124 healthy individuals suggested that plasma hsCRP concentrations had a negative correlation with plasma concentrations of total omega-3 fatty acids, EPA, and docosapentaenoic acid (DPA; C22:5). ${ }^{45}$ In a placebo-controlled, double-blind crossover study of 14 exercise-trained men, 2.2 grams of EPA plus 2.2 grams of DHA daily for 6 weeks reduced hsCRP levels 


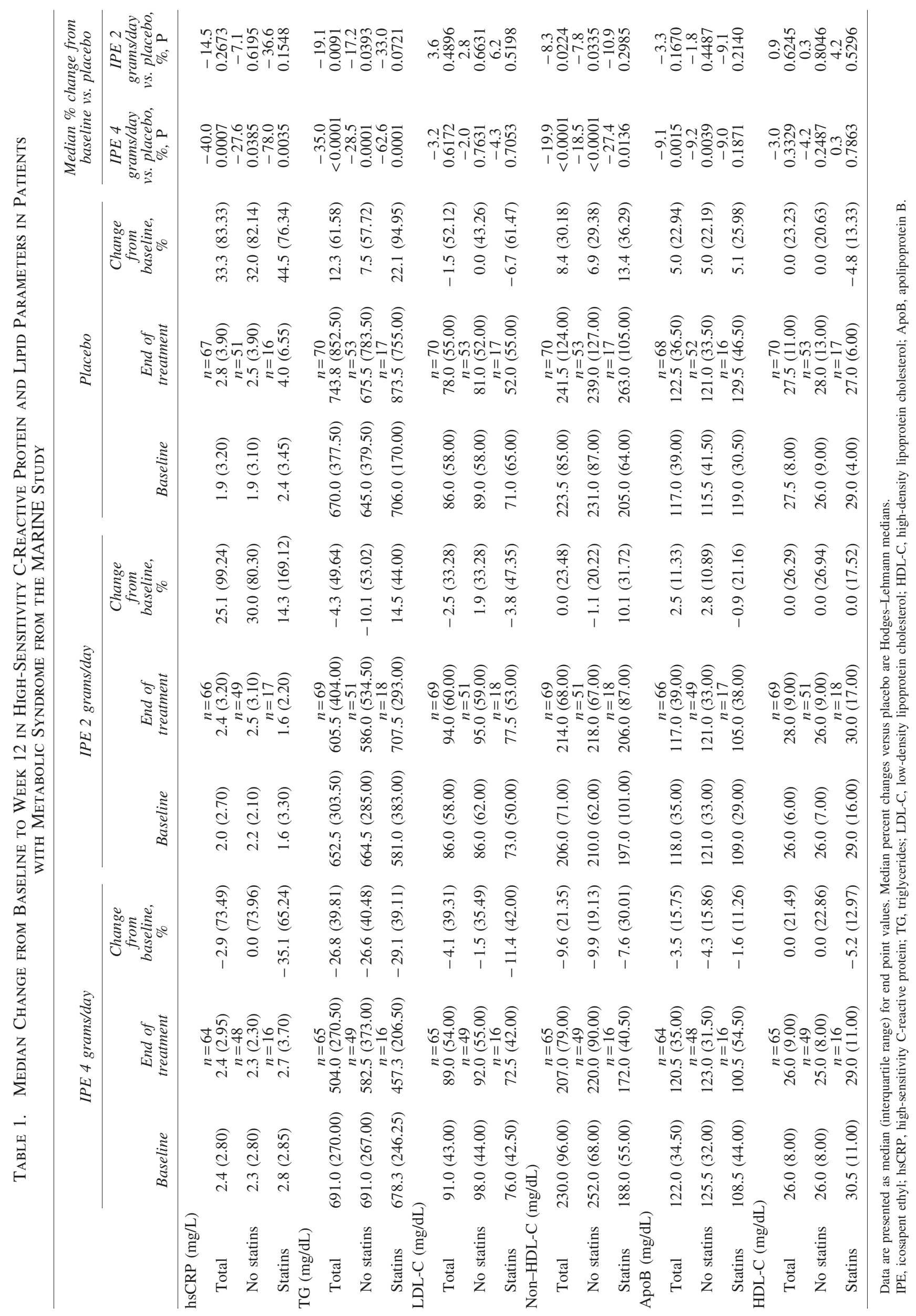



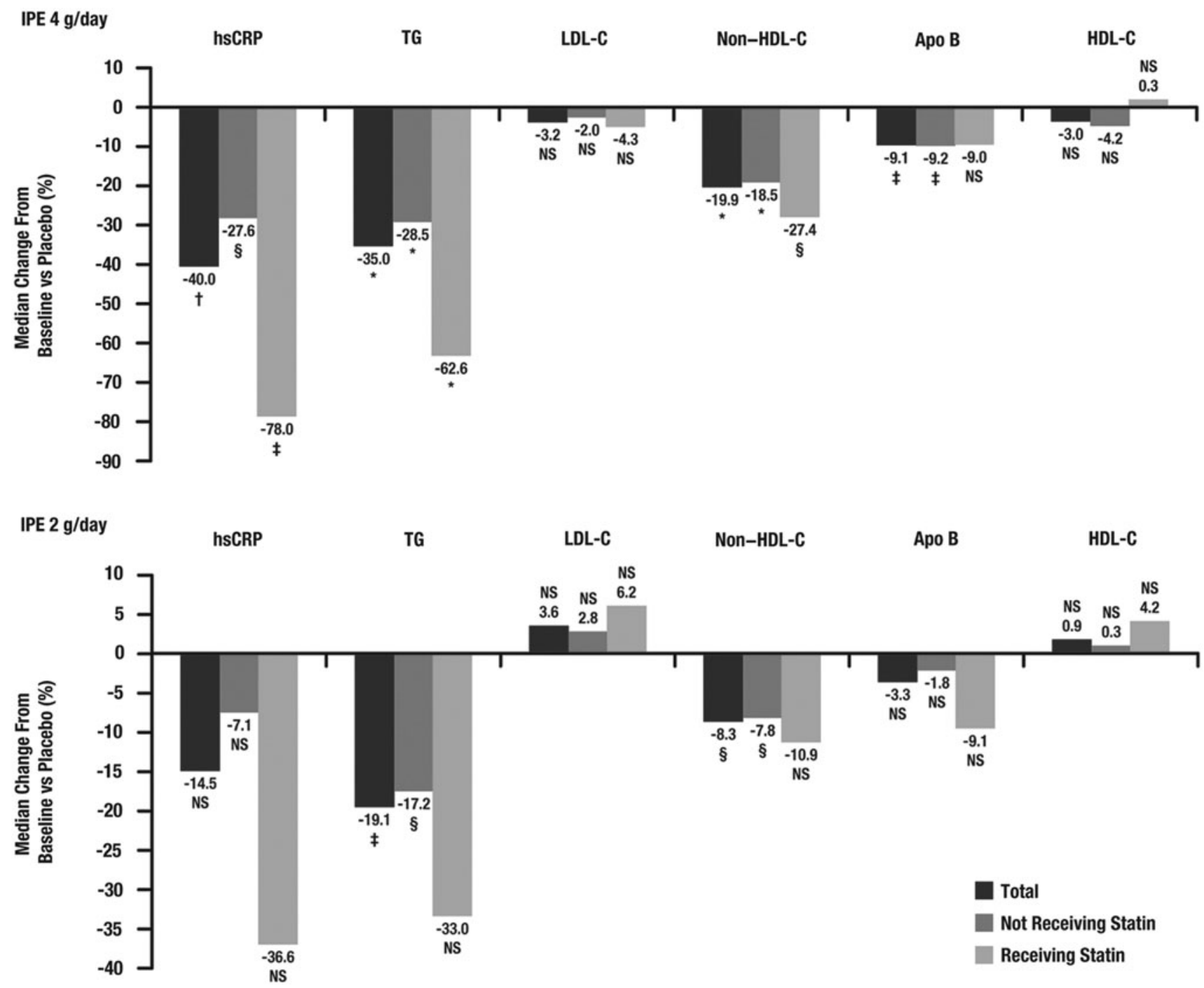

FIG. 1. Median percentage change from baseline to week 12 versus placebo in high-sensitivity C-reactive protein (hsCRP) and lipid parameter levels in patients with metabolic syndrome from the MARINE study. (*) $P \leq 0.0001$; (†) $P<0.001 ;\left({ }^{*}\right) P<0.01 ;\left({ }^{\S}\right) P<0.05$; NS, not significant $(P>0.05)$. IPE, icosapent ethyl; TG, triglycerides; LDL-C, lowdensity lipoprotein cholesterol; HDL-C, high-density lipoprotein cholesterol; ApoB, apolipoprotein B.

compared with placebo $(P<0.05) .{ }^{21}$ In a randomized, doubleblind, placebo-controlled, three-period crossover study, compared with placebo, 8 weeks of 0.85 gram or 3.4 grams daily of an omega-3 fatty acid ethyl ester mixture $\left(\right.$ Lovaza $^{\circledR}$; each 1-gram capsule contained $\sim 0.465$ gram EPA and 0.375 gram DHA) administered to 26 subjects with moderate hypertriglyceridemia (fasting TG levels $150-500 \mathrm{mg} / \mathrm{dL}$ ) did not change hsCRP levels or levels of other inflammatory markers. ${ }^{22}$ In a double-blind, randomized, placebo-controlled study of 34 hypertriglyceridemic men, DHA alone (3 grams/day for 90 days) produced no significant changes in hsCRP levels after 45 days compared with placebo, but reduced hsCRP levels by $15 \%(P<0.05)$ after 90 days of treatment. ${ }^{46}$ Compared with placebo in a double-blind, placebo-controlled study of 59 patients with hypertension and T2DM, DHA or EPA alone (4 grams/day of either treatment for 6 weeks) demonstrated no change in hsCRP levels $(P=0.676){ }^{37}$

Regarding EPA alone, hsCRP levels were unaffected by the intervention with low/moderate-dose EPA combination treatment in a study of 51 Japanese patients with a history of atrial fibrillation who received antiarrhythmic drug treatment for 6 months followed by antiarrhythmic drug treatment plus EPA ethyl ester 1.8 grams/day for 6 months. ${ }^{25}$ However, an earlier study of 44 Japanese patients with metabolic syndrome demonstrated that purified EPA ethyl ester (1.8 grams/day for 3 months as a supplement to dietary therapy) significantly reduced $(P<0.05)$ CRP levels versus before treatment with EPA; statistical significance was not reached versus the control group of diet alone. ${ }^{43}$ In a subsequent study in 92 patients with metabolic syndrome as part of a larger study examining the effects of EPA (1.8 grams/ day) on arterial stiffness, CRP levels were significantly reduced from baseline levels of $1.57 \pm 0.2 \mu \mathrm{grams} / \mathrm{mL}$ to $1.15 \pm 0.2 \mu \mathrm{grams} / \mathrm{mL}$ after 3 months $(P<0.01) .{ }^{24}$ More recently, treatment with 1.8 grams/day EPA was found to significantly reduce hsCRP in a study of 139 Japanese chronic heart failure patients with dyslipidemia versus baseline and versus the no-EPA group (both $P<0.001$ ). ${ }^{47}$

In this current analysis, all patients in ANCHOR were on stable statin therapy prior to study entry. However, the 


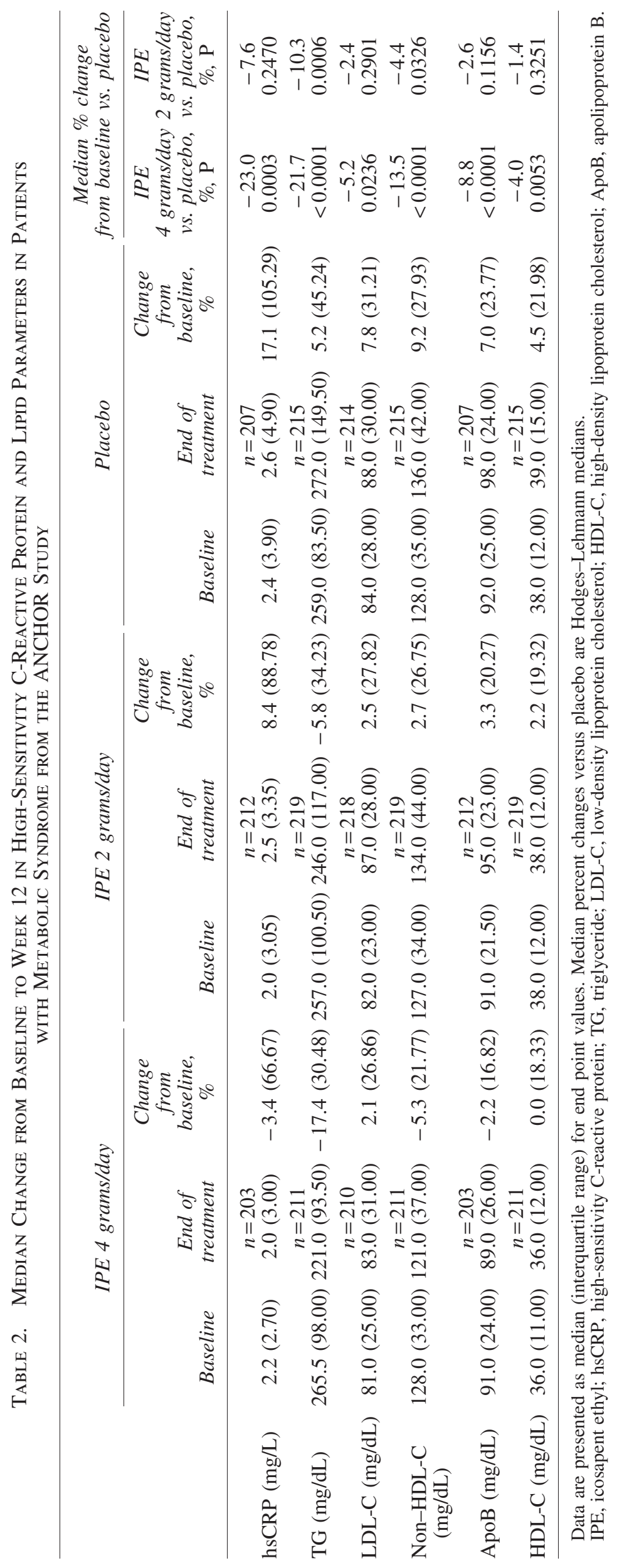




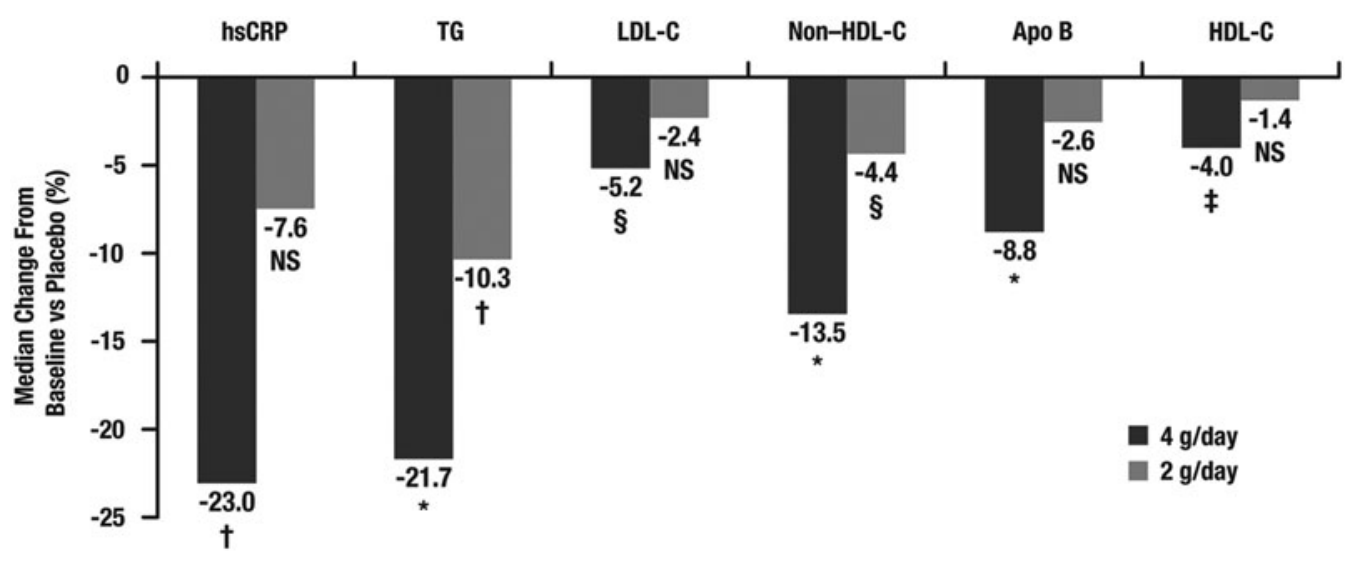

FIG. 2. Median percentage change from baseline to week 12 versus placebo in high-sensitivity C-reactive protein (hsCRP) and lipid parameter levels in patients with metabolic syndrome from the ANCHOR study. (*) $P<0.0001$; (ं) $P<0.001 ;\left(^{*}\right) P<0.01 ;\left(^{\S}\right) P<0.05$; NS, not significant $(P>0.05)$. TG, triglycerides; LDL-C, low-density lipoprotein cholesterol; HDL-C, high-density lipoprotein cholesterol; ApoB, apolipoprotein B.

MARINE study included patients with and without stable statin therapy, allowing for relative comparisons of antiinflammatory and lipid effects. Compared with placebo, among patients with metabolic syndrome in MARINE, IPE treatment produced numerically greater reductions in hsCRP levels among patients receiving stable statin therapy than in those not receiving statin therapy. However, the number of patients in the groups receiving statin therapy was small ( $n=16$ for IPE 4 grams/day, $n=17$ for IPE 2 grams/day, and $n=16$ for placebo), and therefore those results should be interpreted with caution.

As this study evaluated a subset of patients with metabolic syndrome, it is relevant to note the potential effects of omega-3 fatty acids on the components of metabolic syndrome beyond anti-inflammatory effects. ${ }^{48,49}$ When administered as pharmacotherapy, omega-3 fatty acids do not reduce (or increase) body weight. ${ }^{50}$ Thus, it is unlikely that omega-3 pharmacotherapy would significantly alter WC. Although some reports suggest DBP may decrease with high consumption of C20-22 omega-3 fatty acids such as EPA, DPA, and DHA, ${ }^{48,49}$ most controlled, randomized studies of omega-3 fatty acid pharmacotherapies have not produced significant reductions in blood pressure. Regarding glucose, in MARINE, mean changes from baseline in fasting plasma glucose were small and similar in each treatment group; in ANCHOR, there were no significant changes with IPE compared with placebo in fasting plasma glucose. ${ }^{27,28}$

IPE did not increase HDL-C levels. Thus, the only diagnostic component of the metabolic syndrome that substantially changed with IPE was a reduction in TG levels. Other lipid effects often clinically relevant to patients with metabolic syndrome include a reduction in non-HDL-C levels. According to recommendations from some national lipid organizations, non-HDL-C should be considered the primary lipid treatment target, especially among patients with elevated TG levels. ${ }^{51,52}$ In both MARINE and ANCHOR, IPE significantly reduced non-HDL-C levels. ${ }^{27,28}$

Limitations of this report include that it was a post hoc analysis, it used BMI rather than WC as a criterion for metabolic syndrome, and elevated TG levels were a requirement for entry into the clinical trials evaluated. Thus, the metabolic syndrome component of elevated TG levels may be of higher prevalence compared with metabolic syndrome in a general population. It is also unclear whether therapies that lower hsCRP reduce cardiovascular events. Finally, neither the MARINE nor ANCHOR study assessed cardiovascular outcomes. However, the ongoing Reduction of Cardiovascular Events with EPA-Intervention Trial (REDUCE-IT; NCT01492361) is evaluating the effects of IPE 4 grams/day in combination with statin therapy on preventing the occurrence of a first major cardiovascular event in high-risk patients with mixed dyslipidemia. ${ }^{53}$

\section{Conclusions}

Compared with placebo, IPE 4 grams/day improved lipid parameters and reduced hsCRP levels without raising LDL-C levels in statin-treated patients with metabolic syndrome and high ( $\geq 200 \mathrm{mg} / \mathrm{dL}$ and $<500 \mathrm{mg} / \mathrm{dL}$ ) or very high ( $\geq 500 \mathrm{mg} /$ $\mathrm{dL}$ and $\leq 2000 \mathrm{mg} / \mathrm{dL}$ ) TG levels. Taken together, the data suggest that IPE may offer beneficial effects in patients with metabolic syndrome, including those on statin therapy. The hypothesis as to whether these potentially beneficial effects will translate to cardiovascular benefit is being tested in the ongoing cardiovascular outcomes REDUCE-IT study.

\section{Acknowledgments}

Medical writing assistance was provided by Elizabeth Daro-Kaftan, PhD, of Peloton Advantage, Parsippany, NJ, and funded by Amarin Pharma Inc., Bedminster, NJ.

\section{Author Disclosure Statements}

H.E.B.'s research site has received research grants from Alere, Amarin Pharma Inc., Amgen, Ardea, AstraZeneca, Boehringer Ingelheim, Bristol-Myers Squibb, Catabasis, Cymabay, Eisai, Elcelyx, Eli Lilly, Esperion, Forest, Gilead, Given, GlaxoSmithKline, Hanmi, Hisun, High Point Pharmaceuticals LLC, Hoffmann LaRoche, Home Access, Janssen, Merck, Metabolex, Nektar, Novartis, Novo Nordisk, Omthera, Orexigen, Pfizer Inc, Pronova, Regeneron, Sanofi, Takeda, TIMI, Transtech Pharma, Trygg, VIVUS, and WPU Pharmaceuticals. H.E.B. has served as a consultant and/or speaker to Amarin Pharma Inc., Amgen, AstraZeneca, Bristol-Meyers 
Squibb, Catabasis, Daiichi Sankyo, Eisai, Isis, Merck, Novartis, Omthera, VIVUS, and WPU Pharmaceuticals. C.M.B. has received research/grant support from Abbott Diagnostic, Amarin Pharma Inc., Amgen, Eli Lilly, Esperion, Genentech, GlaxoSmithKline, Merck, Novartis, Pfizer Inc, Regeneron, Roche, Roche Diagnostic, and Sanofi-Synthelabo; is a consultant for Abbott Diagnostics, Aegerion, Amarin Pharma Inc., Amgen, Arena, Cerenis, Esperion, Genentech, Genzyme, Kowa, Merck, Novartis, Pfizer Inc, Resverlogix, Roche, and Sanofi-Synthelabo; and has received honoraria from Abbott, Amarin Pharma Inc., AstraZeneca, Bristol-Myers Squibb, Cerenis, Esperion, Genentech, GlaxoSmithKline, Kowa, Merck, Novartis, Omthera, Regeneron, Resverlogix, Roche, and Sanofi-Synthelabo. W.G.S., R.T.D., S.P., and R.A.J. are employees and stock shareholders of Amarin Pharma Inc. S.P. is also affiliated with Chilton Medical Center. R.A.B. and P.N.S. are former employees of Amarin Pharma Inc.

Author contributions: H.E.B., C.M.B., R.A.B., and P.N.S. designed the study. All authors analyzed and interpreted the data and made meaningful contributions to the final manuscript. H.E.B. is guarantor for the article.

\section{References}

1. Bays H. Adiposopathy, "sick fat," Ockham's razor, and resolution of the obesity paradox. Curr Atheroscler Rep 2014; 16:409.

2. Bays HE. Adiposopathy is "sick fat" a cardiovascular disease? J Am Coll Cardiol 2011;57:2461-2473.

3. Bays H. Central obesity as a clinical marker of adiposopathy; increased visceral adiposity as a surrogate marker for global fat dysfunction. Curr Opin Endocrinol Diabetes Obes 2014;21:345-351.

4. Grundy SM, Brewer HB Jr, Cleeman JI, et al. Definition of metabolic syndrome: Report of the National Heart, Lung, and Blood Institute/American Heart Association conference on scientific issues related to definition. Circulation 2004; 109:433-438.

5. Alberti KG, Eckel RH, Grundy SM, et al. Harmonizing the metabolic syndrome: a joint interim statement of the International Diabetes Federation Task Force on Epidemiology and Prevention; National Heart, Lung, and Blood Institute; American Heart Association; World Heart Federation; International Atherosclerosis Society; and International Association for the Study of Obesity. Circulation 2009;120:1640-1645.

6. Bays HE, Toth PP, Kris-Etherton PM, et al. Obesity, adiposity, and dyslipidemia: A consensus statement from the National Lipid Association. J Clin Lipidol 2013;7:304-383.

7. Yang J, Kang J, Guan Y. The mechanisms linking adiposopathy to type 2 diabetes. Front Med 2013;7:433-444.

8. Bays HE. Adiposopathy, diabetes mellitus, and primary prevention of atherosclerotic coronary artery disease: Treating "sick fat" through improving fat function with antidiabetes therapies. Am J Cardiol 2012;110:4B-12B.

9. Grundy SM. Metabolic syndrome pandemic. Arterioscler Thromb Vasc Biol 2008;28:629-636.

10. Bays HE, Gonzalez-Campoy JM, Bray GA, et al. Pathogenic potential of adipose tissue and metabolic consequences of adipocyte hypertrophy and increased visceral adiposity. Expert Rev Cardiovasc Ther 2008;6:343-368.

11. Bays H, Ballantyne C. Adiposopathy: Why do adiposity and obesity cause metabolic disease? Future Lipidol 2006; $1: 389-420$.
12. de Voorde JV, Boydens C, Pauwels B, et al. Perivascular adipose tissue, inflammation and vascular dysfunction in obesity. Curr Vasc Pharmacol 2014;12:403-411.

13. Ridker PM, Kastelein JJ, Genest J, et al. C-reactive protein and cholesterol are equally strong predictors of cardiovascular risk and both are important for quality clinical care. Eur Heart J 2013;34:1258-1261.

14. Kaptoge S, Di AE, Pennells L, et al. C-reactive protein, fibrinogen, and cardiovascular disease prediction. $N$ Engl J Med 2012;367:1310-1320.

15. Harris WS, Miller M, Tighe AP, et al. Omega-3 fatty acids and coronary heart disease risk: Clinical and mechanistic perspectives. Atherosclerosis 2008;197:12-24.

16. Kris-Etherton PM, Harris WS, Appel LJ. Fish consumption, fish oil, omega-3 fatty acids, and cardiovascular disease. Circulation 2002;106:2747-2757.

17. Silva V, Barazzoni R, Singer P. Biomarkers of fish oil omega-3 polyunsaturated fatty acids intake in humans. Nutr Clin Pract 2014;29:63-72.

18. Bays HE, Tighe AP, Sadovsky R, et al. Prescription omega3 fatty acids and their lipid effects: Physiologic mechanisms of action and clinical implications. Expert Rev Cardiovasc Ther 2008;6:391-409.

19. Maki KC, Bays HE, Dicklin MR, et al. Effects of prescription omega-3-acid ethyl esters, coadministered with atorvastatin, on circulating levels of lipoprotein particles, apolipoprotein CIII, and lipoprotein-associated phospholipase A2 mass in men and women with mixed dyslipidemia. J Clin Lipidol 2011;5:483-492.

20. Chan DC, Watts GF, Barrett PH, et al. Effect of atorvastatin and fish oil on plasma high-sensitivity C-reactive protein concentrations in individuals with visceral obesity. Clin Chem 2002;48:877-883.

21. Bloomer RJ, Larson DE, Fisher-Wellman KH, et al. Effect of eicosapentaenoic and docosahexaenoic acid on resting and exercise-induced inflammatory and oxidative stress biomarkers: A randomized, placebo controlled, cross-over study. Lipids Health Dis 2009;8:36.

22. Skulas-Ray AC, Kris-Etherton PM, Harris WS, et al. Doseresponse effects of omega-3 fatty acids on triglycerides, inflammation, and endothelial function in healthy persons with moderate hypertriglyceridemia. Am J Clin Nutr 2011; 93:243-252.

23. Bays HE, Ballantyne CM, Braeckman RA, et al. Icosapent ethyl, a pure ethyl ester of eicosapentaenoic acid: Effects on circulating markers of inflammation from the MARINE and ANCHOR studies. Am J Cardiovasc Drugs 2013;13: 37-46.

24. Satoh N, Shimatsu A, Kotani K, et al. Highly purified eicosapentaenoic acid reduces cardio-ankle vascular index in association with decreased serum amyloid A-LDL in metabolic syndrome. Hypertens Res 2009;32:1004-1008.

25. Watanabe E, Sobue Y, Sano K, et al. Eicosapentaenoic acid for the prevention of recurrent atrial fibrillation. Ann Noninvasive Electrocardiol 2011;16:373-378.

26. Vascepa [package insert]. Bedminster, NJ: Amarin Pharma Inc., 2013.

27. Bays HE, Ballantyne CM, Kastelein JJ, et al. Eicosapentaenoic acid ethyl ester (AMR101) therapy in patients with very high triglyceride levels (from the Multi-center, plAcebo-controlled, Randomized, double-blINd, 12-week study with an open-label Extension [MARINE] trial). Am J Cardiol 2011;108:682-690.

28. Ballantyne CM, Bays HE, Kastelein JJ, et al. Efficacy and safety of eicosapentaenoic acid ethyl ester (AMR101) 
therapy in statin-treated patients with persistent high triglycerides (from the ANCHOR study). Am J Cardiol 2012; 110:984-992.

29. Esser N, Legrand-Poels S, Piette J, et al. Inflammation as a link between obesity, metabolic syndrome and type 2 diabetes. Diabetes Res Clin Pract 2014;105:141-150.

30. Paul A, Ko KW, Li L, et al. C-reactive protein accelerates the progression of atherosclerosis in apolipoprotein E-deficient mice. Circulation 2004;109:647-655.

31. Danenberg HD, Szalai AJ, Swaminathan RV, et al. Increased thrombosis after arterial injury in human C-reactive protein-transgenic mice. Circulation 2003;108:512-515.

32. Ridker PM, Danielson E, Fonseca FA, et al. Rosuvastatin to prevent vascular events in men and women with elevated C-reactive protein. $N$ Engl J Med 2008;359:2195-2207.

33. Zhang D, Che D, Zhao S, et al. Effects of atorvastatin on Creactive protein secretions by adipocytes in hypercholesterolemic rabbits. J Cardiovasc Pharmacol 2007;50:281285.

34. Zhao SP, Zhang DQ. Atorvastatin reduces interleukin-6 plasma concentration and adipocyte secretion of hypercholesterolemic rabbits. Clin Chim Acta 2003;336:103108.

35. Ciubotaru I, Lee YS, Wander RC. Dietary fish oil decreases C-reactive protein, interleukin-6, and triacylglycerol to HDL-cholesterol ratio in postmenopausal women on HRT. J Nutr Biochem 2003;14:513-521.

36. Madsen T, Christensen JH, Blom M, et al. The effect of dietary n-3 fatty acids on serum concentrations of C-reactive protein: A dose-response study. Br J Nutr 2003;89: 517-522.

37. Mori TA, Woodman RJ, Burke V, et al. Effect of eicosapentaenoic acid and docosahexaenoic acid on oxidative stress and inflammatory markers in treated-hypertensive type 2 diabetic subjects. Free Radic Biol Med 2003;35: 772-781.

38. Geelen A, Brouwer IA, Schouten EG, et al. Intake of n-3 fatty acids from fish does not lower serum concentrations of C-reactive protein in healthy subjects. Eur J Clin Nutr 2004;58:1440-1442.

39. Sundrarjun T, Komindr S, Archararit N, et al. Effects of n-3 fatty acids on serum interleukin-6, tumour necrosis factoralpha and soluble tumour necrosis factor receptor p55 in active rheumatoid arthritis. J Int Med Res 2004;32:443454.

40. Sanders TA, Gleason K, Griffin B, et al. Influence of an algal triacylglycerol containing docosahexaenoic acid (22: 6n-3) and docosapentaenoic acid (22: 5n-6) on cardiovascular risk factors in healthy men and women. $\mathrm{Br} J$ Nutr 2006;95:525-531.

41. Madsen T, Christensen JH, Schmidt EB. C-reactive protein and n-3 fatty acids in patients with a previous myocardial infarction: A placebo-controlled randomized study. Eur $J$ Nutr 2007;46:428-430.

42. Murphy KJ, Meyer BJ, Mori TA, et al. Impact of foods enriched with n-3 long-chain polyunsaturated fatty acids on erythrocyte n-3 levels and cardiovascular risk factors. $\mathrm{Br} \mathrm{J}$ Nutr 2007;97:749-757.

43. Satoh N, Shimatsu A, Kotani K, et al. Purified eicosapentaenoic acid reduces small dense LDL, remnant lipoprotein particles, and C-reactive protein in metabolic syndrome. Diabetes Care 2007;30:144-146.

44. Tsitouras PD, Gucciardo F, Salbe AD, et al. High omega-3 fat intake improves insulin sensitivity and reduces CRP and IL6, but does not affect other endocrine axes in healthy older adults. Horm Metab Res 2008;40:199-205.

45. Micallef MA, Munro IA, Garg ML. An inverse relationship between plasma n-3 fatty acids and C-reactive protein in healthy individuals. Eur J Clin Nutr 2009;63:1154-1156.

46. Kelley DS, Siegel D, Fedor DM, et al. DHA supplementation decreases serum C-reactive protein and other markers of inflammation in hypertriglyceridemic men. $J$ Nutr 2009;139:495-501.

47. Kohashi K, Nakagomi A, Saiki Y, et al. Effects of eicosapentaenoic acid on the levels of inflammatory markers, cardiac function and long-term prognosis in chronic heart failure patients with dyslipidemia. J Atheroscler Thromb 2014;21:712-729.

48. Ebbesson SO, Tejero ME, Nobmann ED, et al. Fatty acid consumption and metabolic syndrome components: The GOCADAN study. J Cardiometab Syndr 2007;2:244-249.

49. Lopez-Alvarenga JC, Ebbesson SO, Ebbesson LO, et al. Polyunsaturated fatty acids effect on serum triglycerides concentration in the presence of metabolic syndrome components. The Alaska-Siberia Project. Metabolism 2010; 59:86-92.

50. Bays HE, Maki KC, Doyle RT, et al. The effect of prescription omega-3 fatty acids on body weight after 8 to 16 weeks of treatment for very high triglyceride levels. Postgrad Med 2009;121:145-150.

51. Jacobson TA, Ito MK, Maki KC, et al. National Lipid Association recommendations for patient-centered management of dyslipidemia: Part 1-executive summary. J Clin Lipidol 2014;8:473-488.

52. Bays HE, Jones PH, Brown WV, et al. National lipid association annual summary of clinical lipidology 2015. J Clin Lipidol 2014;8:S1-S36.

53. A study of AMR101 to evaluate its ability to reduce cardiovascular events in high risk patients with hypertriglyceridemia and on statin (REDUCE-IT). Available at http://clinicaltrials.gov/show/NCT01492361/. Accessed January 15, 2015.

Address correspondence to: Harold E. Bays, MD, FTOS, FACC, FACE, FNLA Medical Director/President

Louisville Metabolic and Atherosclerosis Research Center 3288 Illinois Avenue Louisville, KY 40213

E-mail: hbaysmd@aol.com 